\title{
14 Management of Acute Malnutrition in Infants under 6 Months of Age
}

Marko Kerac and Marie McGrath

\section{CONTENTS}

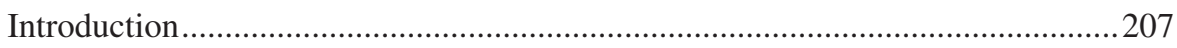

Epidemiology of a "Forgotten Problem" ...........................................................2208

Why Malnutrition Matters for Infants under 6 Months of Age..............................209

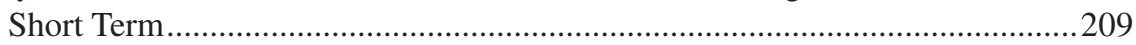

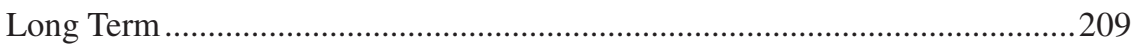

The Challenges of Acute Malnutrition in Infants under 6 Months of Age ............ 210

A Period of Rapid Maturation ........................................................................... 210

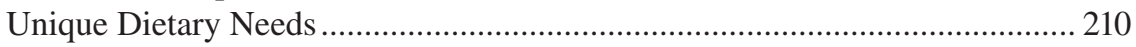

Many and Complex Underlying Causes............................................................ 211

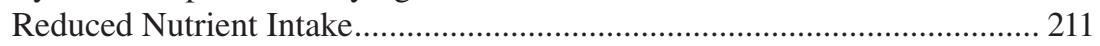

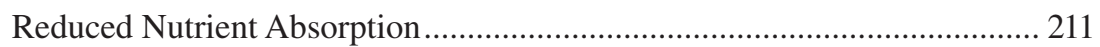

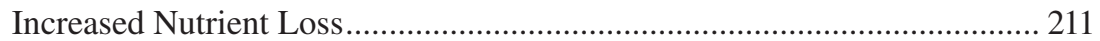

Increased or Impaired Nutrient Utilization ................................................ 212

Assessing Infants under 6 Months of Age ....................................................... 212

Managing Acutely Malnourished Infants under 6 Months of Age ….................... 215

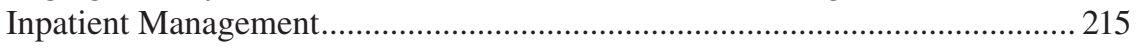



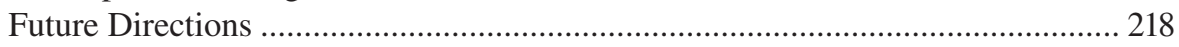

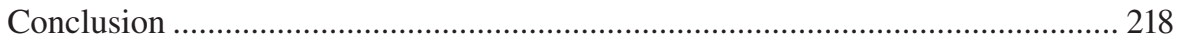

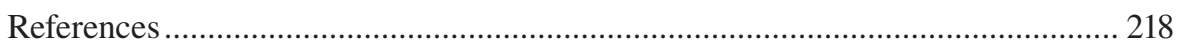

\section{INTRODUCTION}

Both acute malnutrition and nutrition (breastfeeding) in infants under 6 months of age (infants $<6$ months) are important global health issues and have received much international attention over the years. However, it is only recently that the two in combinationthe management of acute malnutrition in infants $<6$ months (MAMI) - have been examined [1]. This chapter outlines the background epidemiology, why acute malnutrition in this age group matters, key challenges around infant $<6$ months malnutrition, current assessment and treatment strategies, and, finally, directions for the future. Readers should look to other chapters of this book for added detail, as 
MAMI has numerous links and synergies with other areas of malnutrition, with many opportunities to benefit both short- and long-term health.

\section{EPIDEMIOLOGY OF A “FORGOTTEN PROBLEM"}

For several decades, it was widely assumed that acute malnutrition in infants $<6$ months was a minor individual-level issue, rather than a significant public health problem. The logical fallacy went like this: Since breastfeeding is associated with good nutritional status, and since infants $<6$ months should be breastfed, poor nutrition among infants $<6$ months must therefore be rare, assuming it only occurs where infants are not breastfed or perhaps where there is early introduction of complementary foods. This was even expressed by authoritative sources, such as the World Health Organization (WHO) "Field Guide to Nutritional Assessment," which stated that "children under six months of age ... are often still breast-fed and therefore satisfactorily nourished" [2]. Combined with the greater practical difficulties of conducting anthropometric measurements in young infants [3,4], this presupposition meant that infants $<6$ months were often omitted from nutrition surveys and surveillance activities [5,6]. As with any problem that is not being actively looked for, acute malnutrition in this age group was often simply overlooked. Specifically, the following factors were overlooked:

- Rates of breastfeeding are almost universally suboptimal [7].

- Despite being the cornerstone of good infant nutrition, breastfeeding is not $100 \%$ protective from nutrition-related problems.

- Nutritional status is dependent on many factors, not just good quality dietary intake [8]. Especially in young infants, there are a large number and variety of health problems that can adversely impact on nutrition. These can be challenging to diagnose and treat, even in high-income, well-resourced settings.

In 2010, in response to questions about infants $<6$ months by field-based practitioners, a report on MAMI [1] and a subsequent research paper [9] aimed to test previous assumptions and quantify the problem as an essential first step toward properly understanding this. An extrapolation of demographic and health survey data from 21 "high burden" low- and middle-income countries found an important burden of disease (Table 14.1). Other observations and issues arising from Table 14.1 include:

- Wasted infants $<6$ months constitute an important proportion of all wasted children aged $<60$ months. This is an argument for program planners and managers needing to take this group seriously and make provisions for their care.

- The 2006 WHO Child Growth Standards (WHO-GS; see Chapter 2 for more detail) really are the gold standard of good growth, setting the bar quite high. Using WHO-GS rather than the previous dominant National Center for Health Statistics (NCHS) growth standards thus results in more infants $<6$ months being recognized as "wasted." 
TABLE 14.1

Global Epidemiology of Wasting in Infants under 6 Months of Age

$\begin{array}{lccc} & \begin{array}{c}\text { All Infants and } \\ \text { Children (0 to 60 } \\ \text { Months), } \boldsymbol{n}=\mathbf{5 5 6} \\ \text { Million }\end{array} & \begin{array}{c}\text { Infants }<\mathbf{6} \text { Months } \\ \text { (WHO Growth } \\ \text { Standards), } \boldsymbol{n}=\mathbf{5 6} \\ \text { Million }\end{array} & \begin{array}{c}\text { Infants }<\mathbf{6} \text { Months } \\ \text { (NCHS Growth } \\ \text { References), } \boldsymbol{n}=\mathbf{5 6} \\ \text { Million }\end{array} \\ \begin{array}{c}\text { Total wasting (millions), } \\ \text { weight-for-length z-score }<-2\end{array} & 58 & 8.5 & 3.0 \\ \begin{array}{c}\text { Moderate wasting (millions), } \\ \text { WLZ } \geq-2 \text { to <-3 }\end{array} & 38 & 4.7 & 2.2 \\ \begin{array}{c}\text { Severe wasting (millions), } \\ \text { WLZ }<-3\end{array} & 20 & 3.8 & 0.8\end{array}$

Source: Adapted from Kerac M, Blencowe H, Grijalva-Eternod C et al., Arch Dis Child 2011, 96(11):1008-13.

- This also challenges some prior assumptions that, since the WHO-GS were based on breastfed infants, they "will result in fewer breastfed babies diagnosed as growing poorly" $[10,11]$.

- Figures for edematous malnutrition are not available. The table thus underestimates the total burden of disease of acute malnutrition and severe acute malnutrition (SAM; edematous malnutrition being part of that case definition) [12]. That said, anecdotal reports suggest that kwashiorkor is uncommon in this age group and that, if bilateral pitting edema is observed, another cause is more likely $[13,14]$.

\section{WHY MALNUTRITION MATTERS FOR INFANTS UNDER 6 MONTHS OF AGE}

\section{ShORT Term}

In the short term, mortality is the most serious risk faced by acutely malnourished infants $<6$ months. Acute malnutrition has a widely recognized, well-described high case fatality rate [15-17], but infants are at particular risk. Reasons include physiological and immunological immaturity, which make them more vulnerable in the first place and more likely to suffer severe adverse consequences. In one recent meta-analysis that compared infants $<6$ months with children 6-60 months in the same treatment programs, the infants' risk of death was significantly greater (risk ratio 1.30, 95\% CI: $1.09,1.56 ; \mathrm{P}<0.01$ ) [18]. Although biologically not unexpected, a key question is how much of this excess mortality can be avoided with improved or alternative treatment.

\section{Long TeRM}

The longer-term effect - and why infant $<6$ months malnutrition is a key topic in this book-is the increasing recognition that early-life nutritional exposures have clinically 
significant long-term "programming" effects on adult health and well being [19,20]. Although the best-known work focuses on exposures during prenatal life [21,22], the window of developmental plasticity (and hence the opportunity to make a positive difference) extends well beyond birth. Optimizing infant nutrition has a major role to play in reducing the current epidemic of noncommunicable disease [23,24]. Acute malnutrition represents an especially severe nutritional "insult" with a high likelihood of correspondingly severe long-term noncommunicable disease (NCD)-related risks [25]. There is a great need for interventions to help infants not only "survive" episodes of malnutrition but also to ultimately "thrive."

\section{THE CHALLENGES OF ACUTE MALNUTRITION IN INFANTS UNDER 6 MONTHS OF AGE}

MAMI currently lags behind great successes in treating older malnourished children [26]. This can be explained by the numerous challenges related to their needs and care.

\section{A Period of Rapid Maturation}

Infants $<6$ months are not simply mini-children; the period represents a major transition from neonatal life, and the beginnings of independence from their mother's milk as the sole source of nutrition.

- Rapid physical and physiological maturation means that a 1-month-old, for example, is very different from a 4-month-old, even though only 3 months separate them in time. What is appropriate for some is not appropriate for all, for example, although exclusive breastfeeding is the target diet for all infants $<6$ months, some acute malnutrition treatment programs report a pragmatic decision to introduce early complementary feeds for those close to 6 months [1].

- There is also a spectrum of development that impacts on care, with some infants maturing faster or slower than most others.

Staff who are skilled and experienced enough to successfully manage these subtleties of approach are often in short supply in settings where malnutrition is common. Any benefits of precisely age-tailored or developmentally tailored treatments thus need to be balanced against the added complexities that these impose on programs; guidelines that are too complex are likely to be poorly implemented in everyday practice. There is also a risk of mixed-messaging regarding feeding practices spilling over to the general population.

\section{Unique Dietary Needs}

Malnourished infants $<6$ months cannot be treated with simple top-up supplementary or therapeutic feeds, as can older malnourished children. Their target diet is exclusive breastfeeding. Even where the mother is around, establishing or reestablishing 
effective exclusive breastfeeding (the mainstay of treatment) is not always straightforward [27-29]. It can require lots of time spent with well-trained, highly skilled support workers. Treatment is thus focused on software (skilled feeding support) rather than hardware (products such as ready-to-use therapeutic food). This often makes this less tangible and, thereby, often also less attractive as an investment for policy makers and program managers.

\section{Many and Complex Underlying Causes}

Rather than a diagnosis in its own right, acute malnutrition is often seen as a symptom of another problem. Only if the underlying cause is properly addressed can the malnutrition be definitively treated. Because the range of causes is greater in young infants (if left untreated, more severe cases can be fatal), assessment and treatment is more complex and, again, more resources are needed.

The underlying cause of infant malnutrition can include several contributing factors.

\section{Reduced Nutrient Intake}

- An insufficient quantity and/or quality offood offered. This can be due to a variety of social/cultural/economic factors. The child might be an orphan, with no wet nurse or a family unable to safely supply a sufficient quality/ quantity of breast milk substitute, or the mother may be working, so is not available to breastfeed sufficiently (this can eventually result in secondary lactation failure).

Although few conditions directly affect the maternal milk supply, many can reduce the frequency/duration of breastfeeding and thus cause secondary lactation failure. It is vital to properly understand the physiology of breastfeeding. More suckling will stimulate more breast milk production; if suckling is reduced for any reason, the breast milk supply will eventually be reduced.

- An anatomical/structural problem. For example, cleft palate, which can affect attachment (feeding suction) and allow milk to enter nasal passages, causing choking.

- A functional problem. For example, poor attachment to the breast due to poor technique; poor suckling or swallowing in prematurity, or cerebral palsy.

- Feed aversion. If an infant is in pain or discomfort when feeding (e.g., in severe reflux or where an anxious carer tries to force-feed), she or he can develop an aversion to feeds, and may try to spit out or otherwise refuse feeds.

\section{Reduced Nutrient Absorption}

- Malabsorption. This can happen in a variety of conditions (e.g., chronic diarrhea, environmental enteric dysfunction, celiac disease, and cystic fibrosis).

\section{Increased Nutrient Loss}

- Vomiting (e.g., severe gastroesophageal reflux or pyloric stenosis). 


\title{
Increased or Impaired Nutrient Utilization
}

- Infections

- Acute (e.g., diarrhea, urinary tract infection)

- Chronic (e.g., HIV, TB)

- Congenital disease (e.g., congenital heart disease)

- Metabolic disorders

The frequency and severity of the underlying problem varies. Breastfeeding attachment problems are, for instance, relatively common; they may not always have major effects on nutritional status, but can be easily and rapidly rectified with skilled support. Congenital metabolic disorders are rare, but can be fatal and difficult to manage, even in resource-rich environments.

It is also vital to note the timing of the nutritional insult; some infants will be small because they were of low birth weight, but are now growing well and "catching up" postnatally. Others will be experiencing postnatal problems.

\section{ASSESSING INFANTS UNDER 6 MONTHS OF AGE}

Definitions of acute malnutrition in infants $<6$ months mirror those in older children, focusing on anthropometry (Table 14.2). This is combined with their clinical status (mainly the presence/absence of integrated management of childhood illness [IMCI] danger signs) to distinguish between complicated acute malnutrition (i.e., sick, clinically high-risk infants requiring more intensive, specialized care available in inpatient facilities) and uncomplicated malnutrition (i.e., clinically stable infants, suitable for outpatient care).

This current case definition has numerous limitations. Refining it is considered to be one of the most urgent future priorities to improve care [30], especially since mislabeling an infant with malnutrition carries serious risks, notably that a breastfeeding mother inappropriately introduces formula feeding, with the consequent risks of infection from unsafe water and contaminated bottles/feeding utensils. This

\author{
TABLE 14.2 \\ Anthropometric Case Definitions of Acute Malnutrition in Infants \\ under 6 Months of Age \\ Term \\ Acute malnutrition (AM) \\ Moderate acute malnutrition (MAM) \\ Severe acute malnutrition (SAM)

\section{Defined By}

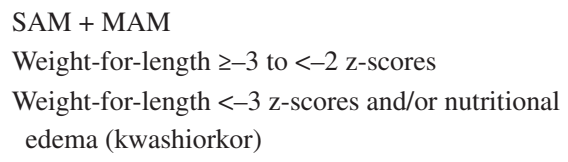


contrasts with the situation for older children, for whom a sensitive (rather than specific) case definition is perfectly acceptable, since the risks of a short period of top-up therapeutic or supplementary food are minimal, hence the benefit-risk balance of intervention is favorable [11].

Other difficulties for infants $<6$ months include:

- Weight-for-length is especially hard to measure in younger infants. Past studies and a recent review highlight poor reliability [3,4,31].

- Weight-for-length cannot be calculated at all for shorter infants $(<45 \mathrm{~cm})$, since the lookup tables do not extend below this level. For these, clinical assessment is carried out.

- There is currently no agreed mid-upper arm circumference (MUAC)-based case definition, as there is for older children. This is a major gap, since MUAC enables quick, effective case finding and is key to the achievement of high coverage by treatment programs [32]. Promising MUAC-based studies do exist, but need replicating in additional settings in order for global policy to change [33].

- Infant $<6$ months malnutrition is special (and thus especially challenging to manage), in that it is critically dependent on the mother. Her prenatal and postnatal nutrition and physical/mental health affect the nutritional status and growth of her infant [34]. Assessing the mother-infant dyad, rather than the infant alone, should be common practice.

- A single anthropometric measurement is difficult to interpret, but sequential growth monitoring-type measures are often not available. Some infants with low weight-for-length will catch up and become "normal" without intervention. Conversely, other infants can be nutritionally vulnerable and not gaining/losing weight, even if their current weight-for-length measurement is still in the "normal" range.

The recent 2013 WHO Guidelines on Managing Acute Malnutrition also note that "infants who have been identified to have poor weight gain and who have not responded to nutrition counselling and support should be admitted for further investigation and treatment" [35].

Recognizing the more complex nature of infant $<6$ months malnutrition, and thus the need for more comprehensive assessment, an IMCI-style assessment checklist has recently been developed (Figure 14.1). This is an attempt to translate the WHO 2013 technical guidelines into an operational handbook for use by field-level health care workers [35]. It also aims to harmonize the WHO malnutrition [35] and IMCI guidelines [36]. Using the IMCI "traffic light" approach (red = urgent problem; yellow = problem but suitable for community-level care; green = no acute problem), it leads the user through key areas, including anthropometry, feeding practice, clinical condition, and maternal well-being. This includes the mother's physical, mental, and nutritional health. 

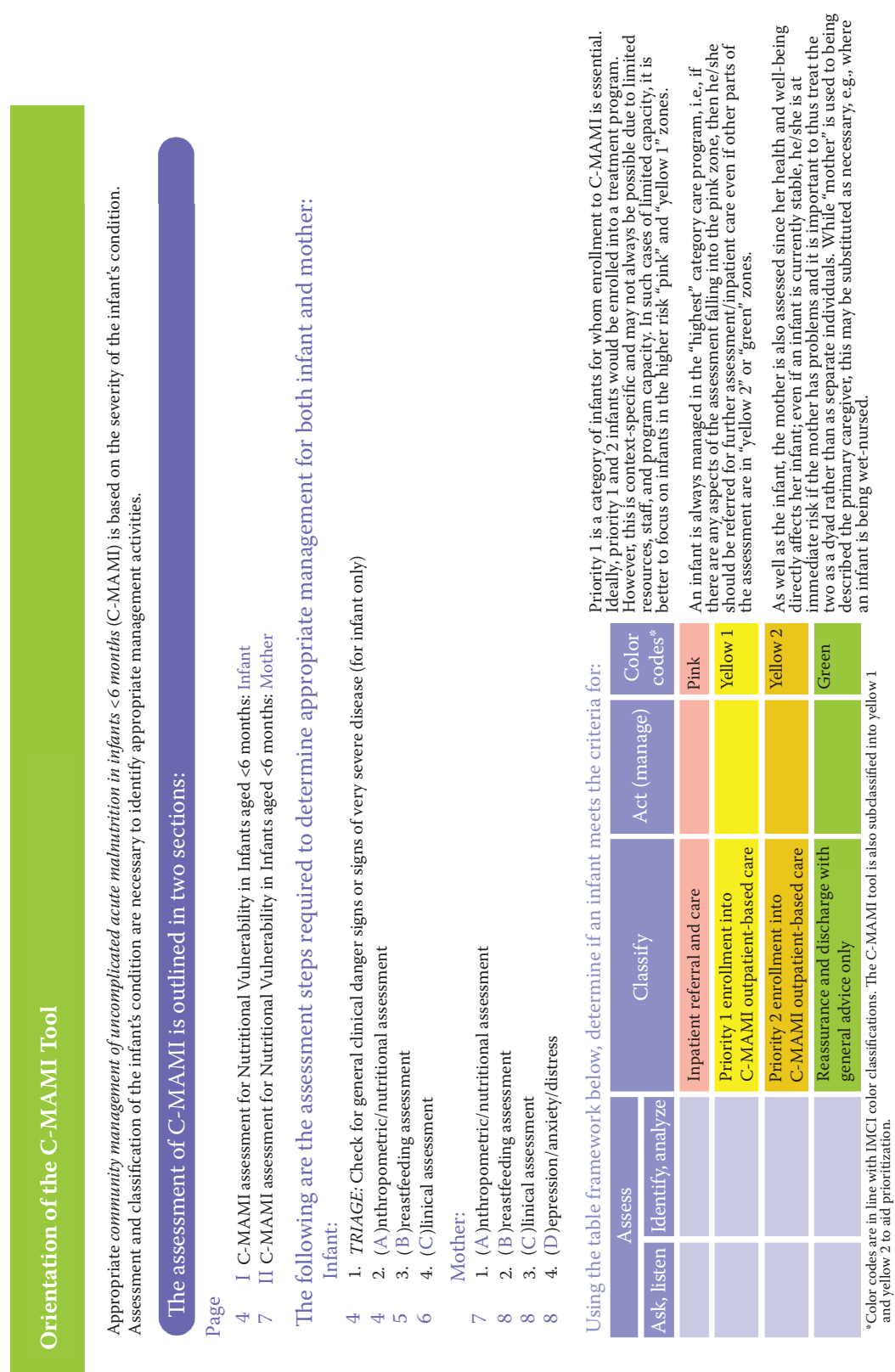

$\dot{0}$
है
हे
z

亩

$\sum_{i}$

吾

莽

.

$\Xi$

들

売

节

莺

衰

苍 


\section{MANAGING ACUTELY MALNOURISHED INFANTS UNDER 6 MONTHS OF AGE}

For many years, normative guidance recommended inpatient care for all acutely malnourished infants $<6$ months [1]. This is still the case in almost all national acute malnutrition guidelines [1]. A major international-level development in the latest 2013 WHO Guidelines on Severe Acute Malnutrition [35] was the introduction of community-based care for infants $<6$ months with "uncomplicated" SAM. This brought their classification and management in line with that of older children [26].

Key principles that guide management include:

- Early identification of malnourished and nutritionally vulnerable infants in the community, and at any health-related contact point (e.g., when attending for immunizations, or when seeking clinic or hospital treatment for an acute or chronic illness).

- Early treatment in the community wherever possible (uncomplicated cases).

- Early referral and treatment of sick infants (complicated cases).

- Breastfeeding support to restore effective exclusive breastfeeding wherever possible.

- Careful use of breast milk substitutes for nonbreastfed infants, if wetnursing or banked breast milk is not available.

- General medical care for any underlying problems.

- All severely malnourished infants, including those with uncomplicated diseases, should receive a course of broad-spectrum antibiotics (oral if possible, parenteral if needed).

- Other conditions (e.g., HIV, sepsis, surgical problems, or disability) should be dealt with as needed.

- Follow-up on infants postdischarge in the community.

- Targeted support to the mother that considers her nutritional, physical, and mental condition.

- Considering and engaging with the broader household and community and societal influences on the mother-infant dyad that may affect feeding and/ or care practices.

\section{InPATIENT Management [37]}

The cornerstone of inpatient care is supplementary suckling. This aims to restore exclusive breastfeeding, and involves taping a small nasogastric tube onto the breast, with the tip adjacent to the nipple. Supplementary milk (infant formula, F-75 therapeutic milk, or diluted F-100 therapeutic milk-there is no hard evidence as to which of these is best) [36] is given via the tube. Crucially, however, the infant is placed on the breast, and attempts to suckle at the same time, thereby stimulating breast milk production. As the quantity of breast milk supply begins to increase, the quantity of supplementary milk can be decreased and, eventually, stopped entirely. A galactagogue (e.g., metoclopramide) can be given to aid this process. 
Although supplementary sucking can be a great success in centers with experience, success rates vary [1] and depend on many factors, including motivation, breastfeeding views, practicalities, understanding, and perceptions of hospital-based medicine [27].

The management of nonbreastfed infants is not well catered for in terms of guidance, and creates huge practical challenges in resource-poor settings. It begins by feeding infants with the same supplementary milks (infant formula, F-75, or diluted F-100) to get them back to their normal weight. The biggest difficulty then comes in terms of what to do longer term. Identifying a wet nurse is a priority when culturally acceptable and available. Where wet nursing is not viable, infant formula is necessary for infants $<6$ months; nutritional indication for infant formula in children 6 months to 2 years depend on the complementary foods available, in particular animal source foods [38]. Where infant formula is not a viable option, a 5-month-old nonbreastfed infant recovering from malnutrition might start early complementary feeds based on skilled assessment as part of a pragmatic care approach, but a 2- or 3-month-old needs a longer term appropriate breast milk substitute, which, in many settings, may not be affordable or available, and requires a package of support services, including water, sanitation, and hygiene (WASH), and access to nutrition and health monitoring. The risks of relapse and of chronic undernutrition in these infants are high. More evidence is needed as to what works for this especially vulnerable group of infants and their carers.

\section{Outpatient Management}

The WHO 2013 guidance recommends the following for the outpatient care of infant $<6$ months with severe acute malnutrition [35]:

- "Counselling and support for optimal infant and young child feeding, based on general recommendations for feeding infants and young children, including for low birth weight infants,

- Weekly weight monitoring to observe changes,

- Referral into inpatient care if the infant does not gain weight, or loses weight, and

- Assessment of the physical and mental health status of mothers or caregivers with appropriate treatment and support provided."

Since these are all low-risk interventions, it is appropriate to extend the same approach to infants with moderate acute malnutrition and even those who are nutritionally vulnerable but do not (yet) have weight-for-length $<-2$ z-scores. Again, the C-MAMI tool brings together and reformats guidance that has been successful in other settings for other infant populations. Figure 14.2 is an example from the C-MAMI tool of how to identify and address poor attachment, which is a common problem. 

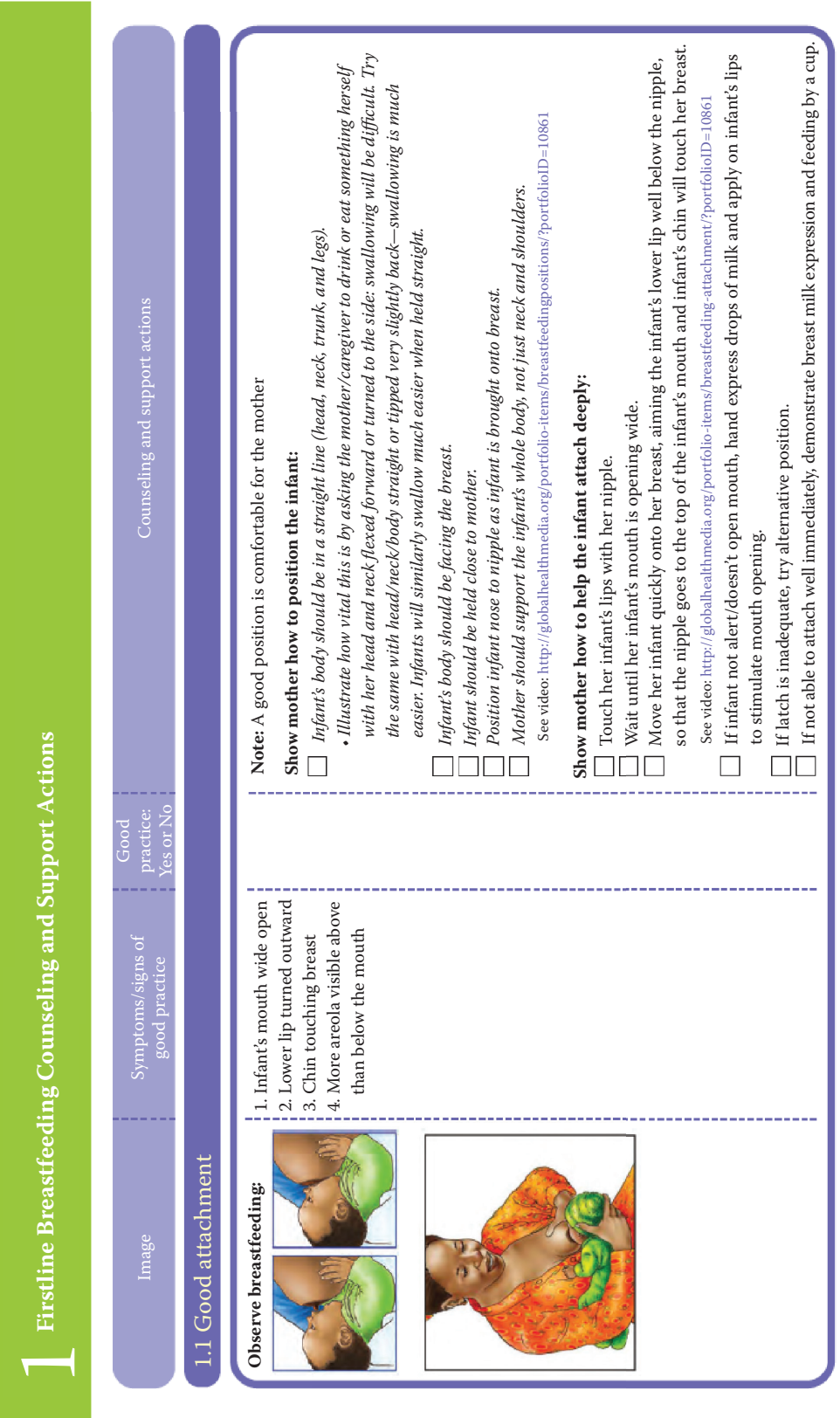

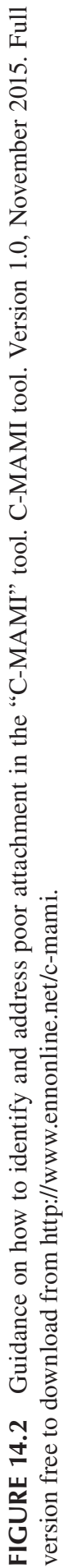




\section{FUTURE DIRECTIONS}

Most WHO recommendations for managing infants $<6$ months are "strong" recommendations [35]. It is vital, however, to acknowledge that the underlying evidence is "low quality" [39]. This represents a problem but also an opportunity to rapidly move things forward for this vulnerable group.

In a recent research prioritization exercise [30], the three top-ranked research questions were

1. How should infant $<6$ months SAM be defined? As discussed, there are serious problems with the current anthropometry weight-for-length focused case definition of acute malnutrition in infants $<6$ months. The need for a refined and improved case definition offers a great opportunity to embrace more holistic terms of reference, assessing infants and their mothers and their social/home environments. Only thus can their problems be understood, and truly effective care packages planned and put in place for each individual.

2. What are/is the key opportunities/timing when infant SAM management can be incorporated with other health care programs? MAMI offers an opportunity to connect numerous emergency and development programs and stakeholders across many sectors. Rather than being a narrow vertical program, it has to ensure links with other health and social programs, so that every occasion where there is contact with an infant provides an opportunity for identifying those who are nutritionally vulnerable and supporting/treating them.

3. What are the priority components of a package of care for outpatient treatment of infant $<6$ months SAM? MAMI requires interventions at many levels, from individual case management to societal level interventions, such as social protection/maternity pay.

\section{CONCLUSION}

MAMI is a new and rapidly evolving area. Although much progress has been made, much still needs to take place. The 2013 WHO guidelines on infant $<6$ months are a major step forward, but still need to be translated into country-level guidelines and, most important, to front-line clinical settings. Despite the many challenges and difficulties, addressing MAMI offers an opportunity to escape from siloed nutrition thinking and programming. Strengthening the early identification and management of nutritionally vulnerable infants has to go past nutrition and involve other sectors, especially reproductive health, neonatal health, and WASH; has to include the nutrition and health of mothers and, within that, adolescents; and has to innovate and look to development programming for management strategies.

\section{REFERENCES}

1. Emergency Nutrition Network/University College London/Action Contre la Faim. Management of Acute Malnutrition in Infants (MAMI) project. Available at: http:// www.ennonline.net/mamitechnicalreview (accessed February 5, 2017). 
2. World Health Organization. Field guide on rapid nutritional assessment in emergencies. Geneva: World Health Organization; 1995.

3. Mwangome MK, Berkley JA. The reliability of weight-for-length/height z scores in children. Matern Child Nutr 2014; 10(4):474-80.

4. Mwangome M, Berkley J. Measuring infants aged below 6 months: Experience from the field. ENN Field Exchange 2014; 47:34.

5. Prudohn, C. Including infants in nutrition surveys. ENN Field Exchange 2000; 9:15.

6. Lopriore C, Dop MC, Solal-Celigny A et al. Excluding infants under 6 months of age from surveys: Impact on prevalence of pre-school undernutrition. Public Health Nutr 2007; 10(1):79-87.

7. Victora CG, Bahl R, Barros AJ et al. Breastfeeding in the 21st century: Epidemiology, mechanisms, and lifelong effect. Lancet 2016; 387(10017):475-90.

8. Goh LH, How CH, Ng KH. Failure to thrive in babies and toddlers. Singapore Med J 2016; 57(6):287-91.

9. Kerac M, Blencowe H, Grijalva-Eternod $\mathrm{C}$ et al. Prevalence of wasting among under 6-month-old infants in developing countries and implications of new case definitions using WHO growth standards: A secondary data analysis. Arch Dis Child 2011; 96(11):1008-13.

10. Waterlow JC. Protein-energy malnutrition. 2nd ed. London: Edward Arnold; 1992.

11. Ahmad UN, Yiwombe M, Chisepo P et al. Interpretation of World Health Organization growth charts for assessing infant malnutrition: A randomised controlled trial. J Paediatr Child Health 2014; 50(1):32-39.

12. Frison S, Checchi F, Kerac M. Omitting edema measurement: How much acute malnutrition are we missing? Am J Clin Nutr 2015; 102(5):1176-81.

13. Karunaratne R. Management of acute malnutrition in Infants aged $<6$ months (MAMI) in Malawi: Prevalence and risk factors in an observational study. Royal College of Paediatrics \& Child Health Annual Meeting 2015. Birmingham, UK.

14. Emergency Nutrition Network. Edema in infants $<6$ months and PLW [Internet]. Accessible at: http://www.en-net.org/question/1659.aspx (accessed February 5, 2017).

15. Black RE, Victora CG, Walker SP et al. Maternal and child undernutrition and overweight in low-income and middle-income countries. Lancet 2013; 382(9890):427-51.

16. Schofield C, Ashworth A. Why have mortality rates for severe malnutrition remained so high? Bull World Health Organ 1996; 74(2):223-9.

17. Heikens GT. How can we improve the care of severely malnourished children in Africa? PLoS Med 2007; 4(2):e45.

18. Grijalva-Eternod CS, Kerac M, McGrath M et al. Admission profile and discharge outcomes for infants aged less than 6 months admitted to inpatient therapeutic care in 10 countries. A secondary data analysis. Matern Child Nutr 2016 (in press) doi: 10.1111 /men.12345.

19. Ruemmele FM. Early programming effects of nutrition: Life-long consequences? Ann Nutr Metab 2011; 58(Suppl 2):5-6.

20. Tarry-Adkins JL, Ozanne SE. Mechanisms of early life programming: Current knowledge and future directions. Am J Clin Nutr 2011; 94(6):S1765-71.

21. Fernandez-Twinn DS, Ozanne, SE. Early life nutrition and metabolic programming. Ann N Y Acad Sci 2010; 1212:78-96.

22. Eriksson JG. Early programming of later health and disease: Factors acting during prenatal life might have lifelong consequences. Diabetes 2010; 59(10):2349-50.

23. Lanigan J, Singhal A. Early nutrition and long-term health: A practical approach. Proc Nutr Soc 2009; 68(4):422-9.

24. Singhal A. The global epidemic of noncommunicable disease: The role of early-life factors. Nestle Nutr Inst Workshop Ser 2014; 78:123-32. 
25. Lelijveld N, Seal A, Wells JC et al. Chronic disease outcomes after severe acute malnutrition in Malawian children (ChroSAM): A cohort study. Lancet Glob Health 2016; 4(9):e654-62.

26. Trehan I, Manary MJ. Management of severe acute malnutrition in low-income and middle-income countries. Arch Dis Child 2015; 100(3):283-7.

27. Lelijveld N, Mahebere-Chirambo C, Kerac M. Carer and staff perspectives on supplementary suckling for treating infant malnutrition: Qualitative findings from Malawi. Matern Child Nutr 2014; 10(4):593-603.

28. Lutter CK, Morrow AL. Protection, promotion, and support and global trends in breastfeeding. Adv Nutr 2013; 4(2):213-9.

29. Sinha B, Chowdhury R, Sankar MJ et al. Interventions to improve breastfeeding outcomes: A systematic review and meta-analysis. Acta Paediatr 2015; 104(467):114-34.

30. Angood C, McGrath M, Mehta S et al. Research priorities to improve the management of acute malnutrition in infants aged less than six months (MAMI). PLoS Med 2015; 12(4):e1001812.

31. Mwangome MK, Fegan G, Mbunya R et al. Reliability and accuracy of anthropometry performed by community health workers among infants under 6 months in rural Kenya. Trop Med Int Health 2012; 17(5):622-9.

32. Myatt M, Khara T, Collins S. A review of methods to detect cases of severely malnourished children in the community for their admission into community-based therapeutic care programs. Food Nutr Bull 2006; 27(3 Suppl):S7-23.

33. Mwangome MK, Fegan G, Fulford T et al. Mid-upper arm circumference at age of routine infant vaccination to identify infants at elevated risk of death: A retrospective cohort study in the Gambia. Bull World Health Organ 2012; 90(12):887-94.

34. Stewart RC. Maternal depression and infant growth: A review of recent evidence. Matern Child Nutr 2007; 3(2):94-107.

35. World Health Organization. Updates on the management of severe acute malnutrition in infants and children. Geneva: World Health Organization; 2013.

36. World Health Organization. Integrated management of childhood illness (IMCI) chart booklet. Geneva: World Health Organization; 2014.

37. Kerac M, Tehran I, Lelijveld $\mathrm{N}$ et al. Inpatient treatment of severe acute malnutrition in infants aged $<6$ months. Geneva: World Health Organization; 2012.

38. World Health Organization. Guiding principles for feeding non-breastfed children 6-24 months of age. Geneva: World Health Organization; 2003.

39. Guyatt GH, Oxman AD, Vist GE et al. GRADE: An emerging consensus on rating quality of evidence and strength of recommendations. BMJ 2008; 336(7650):924-6. 